\title{
The Supernova Relic Neutrino Backgrounds at KamLAND and Super-Kamiokande
}

\author{
Louis E. Strigari ${ }^{1}$, Manoj Kaplinghat ${ }^{3}$, Gary Steigman ${ }^{1,2}$, and \\ Terry P. Walker ${ }^{1,2}$ \\ ${ }^{1}$ Department of Physics, The Ohio State University, Columbus, OH 43210 \\ ${ }^{2}$ Department of Astronomy, The Ohio State University, Columbus, OH 43210 \\ ${ }^{3}$ Department of Physics, University of California, Davis, CA 95616 \\ E-mail: strigari@mps.ohio-state.edu, kaplinghat@physics.ucdavis.edu, \\ steigman@mps.ohio-state.edu, and twalker@mps.ohio-state.edu
}

\begin{abstract}
We calculate the Supernova Relic Neutrino (SRN) background flux for the KamLAND and Super-Kamiokande (Super-K) detectors, motivated by the reduction in background at Super-K and new results for the star formation history (e.g., from the Sloan Digital Sky Survey (SDSS)). Our best estimate for the flux at Super-K is slightly below, but very close to the current Super-K upper limit. The Super-K upper limit is already inconsistent with a range of star formation histories allowed by the SDSS data. We estimate that the SRN background should be detected (at $1 \sigma$ ) at Super-K with a total of about 9 years (including the existing 4 years) of data. While KamLAND is a much smaller detector compared to Super-K, it profits from being practically background-free and from its sensitivity to the lower energy supernova neutrinos. KamLAND could make a $1 \sigma$ detection of the SRN with a total of about 5 years of data. Given the small expected SRN event rate, we also consider the detection of the SRN in a modified Super-K detector with a lower threshold and reduced background where the time to detection can be reduced by a factor of 10 relative to the existing Super-K estimate.
\end{abstract}




\section{Introduction}

In its death throes as a type II supernova ( $\mathrm{SN}$ II $)$ a massive star $\left(\gtrsim 8 \mathrm{M}_{\odot}\right)$ ends its life emitting $\sim 99 \%$ of its energy, $\sim 10^{53} \mathrm{ergs}$, in neutrinos. This theoretical expectation was spectacularly confirmed by the detection of a handful of neutrinos from SN1987A in the nearby Large Magellanic Cloud [1, 2]. While the much larger, more heavily instrumented detectors such as Super-Kamiokande (Super-K) and KamLAND await the flood of neutrinos from another such nearby SN II, it is interesting to ask if either detector might observe the diffuse, isotropic flux of neutrinos from all SN in events that have ever occurred within the observable universe.

The detection of this cosmological background of core-collapse supernova relic neutrinos (SRN) offers a new probe of SN II neutrino physics and of the high redshift Universe. The basic picture of core-collapse SN II could be tested, not only locally but also at high redshifts $(z \gtrsim 1)$. From the SRN we will also obtain estimates of the supernova rate (proportional to the star formation rate for $M \gtrsim 8 \mathrm{M}_{\odot}$ ) and of the metal enrichment rate which are completely independent of those from optical and UV surveys. Note that other tracers of the cosmological star formation history typically only lead to lower bounds on the star formation rate. Comparison with traditional methods would yield information about high redshift star formation complementary to that from future high redshift galaxy surveys like DEEP2 3] (which targets $z \lesssim 1$ ). Here we consider the possibility of SRN detection at two existing neutrino detectors, Super-K and KamLAND. We also consider the detection of the SRN in a modified Super-K detector with lower threshold and reduced backgrounds (like the recently proposed SK-GADZOOKS [4]). As discussed later, detection of the SRN at both KamLAND and Super-K can be used to probe the cosmic star formation history at $z \gtrsim 1$, of which little is known from traditional astronomical methods.

The prediction of the SRN flux has been the subject of many previous investigations [5]. The earlier study of Kaplinghat, Steigman, and Walker 2000 (KSW) 6] reached the pessimistic conclusion that it would be unlikely for Super-K to detect the SRN background, a result driven by three factors. First there was the realization that for Super-K to detect these relic neutrinos, the actual flux must be close to the predicted upper bound and, in addition, the $\bar{\nu}_{\mathrm{e}}$ must be nearly maximally mixed with $\bar{\nu}_{\mu}$ or $\bar{\nu}_{\tau}$. However, as discussed below, recent results on the cosmic star formation history from observational data, including those from the Sloan Digital Sky Survey (SDSS), lead to a predicted SRN rate that is closer to the upper bound derived in KSW. Furthermore, neutrino experiments, such as SNO, KamLAND, and Super-K have clearly identified the large mixing angle case as the preferred solution to the solar neutrino problem [7, 8, implying that the $\mathrm{SN}$ is $\bar{\nu}_{e}$ are (nearly) maximally mixed. The second factor concerned the backgrounds to the detection of the SRN. KSW estimated the primary background at Super-K, due to sub-Cherenkov muons (to be discussed later), by 
extrapolating from older Kamiokande data. The estimated background to the detection of the SRN in the energy window (for positrons) from 19 to $35 \mathrm{MeV}$ was 39 events per year. The latest Super-K analysis [9] reveals that the background in the same energy window is actually about 19 events per year. The third factor leading to the negative conclusion of KSW concerned the spectrum of the SRN flux. The difference between the spectra of the SRN and the sub-Cherenkov muon neutrino fluxes could help to separate the SRN signal from this background but, given the large expected background and the uncertainties in the supernova rate, KSW did not attempt to account for this in their analysis. However, considering the current status of the first two factors, it is clear that a more detailed analysis is now appropriate. Although with 4 years of data Super-K did not see any supernova relic neutrinos, they did reduce the upper limit on the SRN flux at $90 \%$ C.L. to $1.2 \bar{\nu}_{\mathrm{e}} \mathrm{cm}^{-2} \mathrm{~s}^{-1}$, for positron energies $>18 \mathrm{MeV}[9$. This limit is more than two orders of magnitude smaller than the previous Kamiokande-II upper limit [10] and is approaching several of the theoretical estimates of the SRN flux. Given this very encouraging state of affairs, along with the advances in recent years in our understanding of the cosmic star formation history, we believe it is timely to attempt a realistic, quantitative estimate of the likely range for the SRN flux.

For Super-K our analysis is restricted to positron energies $>18 \mathrm{MeV}$. In contrast the KamLAND detector, as a result of their detection technique, have much smaller backgrounds at energies suitable for detecting SRN and are therefore sensitive to much lower energy neutrinos (positrons with $\mathrm{E}>6 \mathrm{MeV}$ ). Thus, even though KamLAND is a much smaller detector than Super-K, it profits with respect to Super-K by being sensitive to more of the SRN spectrum and also by its sensitivity to neutrinos from supernovae at higher redshifts compared to Super-K. We also present results for the SRN flux expected at KamLAND in the idealized limit in which KamLAND is able to utilizes its entire 1 kton volume for detecting SRN with $100 \%$ efficiency. We also discuss detection in the hypothetical SKGADZOOKS detector, the propeties of which were described in Beacom \& Vagins 4. Estimates for a hypothetical detector "HyperKamiokande" [11, with a fiducial volume of $V \approx 890 \mathrm{kton}\left(\approx 40 V_{\mathrm{SK}}\right)$, can be obtained by appropriately scaling the flux at Super-K and noting that the errors on the flux scale as $V^{-1 / 2}$.

\section{Detectors}

Super-K is a 22.5 kton fiducial mass water Cerenkov detector which can detect SRNs via inverse beta decay $\bar{\nu}_{\mathrm{e}}+\mathrm{p} \rightarrow \mathrm{e}^{+}+\mathrm{n}$. Below $18 \mathrm{MeV}$ spallation events resulting from the interaction of cosmic ray muons with oxygen nuclei form the primary background at Super$\mathrm{K}$ [12]. Above $18 \mathrm{MeV}$ there are two primary backgrounds. The first comes from low energy atmospheric $\bar{\nu}_{\mathrm{e}}$ produced from the decay chain of $\pi^{ \pm}$and $\mu^{ \pm}[13]$. Below $25 \mathrm{MeV}$ the 
SRN flux from our median estimate (see below), which peaks at roughly $5 \mathrm{MeV}$, exceeds the atmospheric $\bar{\nu}_{\mathrm{e}}$ flux. The second background comes from atmospheric $\nu_{\mu}$ which interact with a nucleus to form a $\mu^{ \pm}$with kinetic energy $\leq 53 \mathrm{MeV}$. Such $\mu$ s are not detected as they are below the threshold for Cerenkov radiation, but their $e^{ \pm}$decay products are detected, with energy distribution given by the Michel spectrum. By performing a multicomponent fit to the observed Michel spectrum, as well as that for the atmospheric $\nu$, Super-K obtained their impressive limit on the SRN background flux [9]. It would be ideal if there were a way to detect the recoil neutrons from the $\bar{\nu}_{\mathrm{e}}-p$ interaction (analgous to the neutral current detection at SNO) as was suggested in a recent proposal [4]. This neutron tagging would remove the $\nu_{\mu}$ and spallation backgrounds, allowing Super-K to move to a lower energy threshold of $10 \mathrm{MeV}$. Such a reduction in threshold will tell us more about high redshift supernovae and the corresponding star formation history than any other existing method. Regardless, for the estimates of the SRN flux at Super-K presented here we limit ourselves to neutrinos with energies $>19.3 \mathrm{MeV}$ (positrons with $\mathrm{E}_{e^{+}}>18 \mathrm{MeV}$ ). The current predictions from Ando, Sato, \& Totani [5] show that for SK the expected flux is roughly $0.3 \mathrm{~cm}^{-2} \mathrm{~s}^{-1}$, which is close to the lower limit derived from Fukugita \& Kawasaki [5]. Below we show how median estimates of the SN rate as a function of redshift can give a flux very close to the current Super-K upper limit. The corresponding event rate of the SRN at Super-K in this range is expected to be 1 to 2 per year, and a few more years of data are likely to yield a positive detection.

KamLAND is a 1 kton liquid scintillation detector designed to search for evidence of $\bar{\nu}_{\mathrm{e}}$ oscillations utilizing $\bar{\nu}_{\mathrm{e}}$ from nuclear power reactors 114, 15, 7]. KamLAND detects $\bar{\nu}_{\mathrm{e}}$ via inverse beta decay by a prompt signal from positron annihilation, followed by a $\sim 200 \mu \mathrm{s}$ time-delayed neutron capture $\gamma$-ray of $2.2 \mathrm{MeV}$. The spectrum of the reactor $\bar{\nu}_{\mathrm{e}}$ peaks near $3 \mathrm{MeV}$, and with neutrino oscillations is negligible above $6 \mathrm{MeV}$. The KamLAND collaboration analysis imposes a lower cutoff of $2.6 \mathrm{MeV}$ to account for the background from terrestrial $\bar{\nu}_{\mathrm{e}}$ sources. Unlike Super-K, KamLAND can readily exclude the invisible muon decay background since the prompt signal from positron annihilation is not followed by neutron capture. The KamLAND background at energies greater than $6 \mathrm{MeV}$ is from atmospheric $\bar{\nu}_{\mathrm{e}}$, which competes with our estimated SRN signal at energies $\gtrsim 25 \mathrm{MeV}$. KamLAND's smaller background at low energies, in the region where the SRN spectrum peaks, may make it possible for KamLAND to detect the SRN background. Preliminary results at KamLAND for a 0.28 kton-year exposure show no $\bar{\nu}_{\mathrm{e}}$ signal above the small expected backgrounds [16]. Current theoretical estimates assuming that KamLAND can use the entire 1 kton volume for SRN detection (Ando, Sato and Totani 2003 [5]) suggest that in the energy window $10-25 \mathrm{MeV}$, the $\mathrm{SRN}$ event rate is $\sim 0.1 \bar{\nu}_{\mathrm{e}} \mathrm{yr}^{-1}$. In our analysis here the SRN flux for (positron) energies $\mathrm{E}>6 \mathrm{MeV}$ is considered. This lower energy threshold is chosen since, so far, there are no events seen above this energy at 
KamLAND [7]. In addition, in our estimates for KamLAND we adopt an idealization in which KamLAND utilizes the entire 1 kton fiducial volume with $100 \%$ efficiency for SRN. Based on our best estimate of the supernova rate which saturates the Super-K bound, the expected rate in an idealized KamLAND detector is 0.4 events per year.

\section{Flux of the SRN}

The differential flux of SRN $\bar{\nu}_{\mathrm{e}}, d F / d E$, depends on the magnitude and evolution (as a function of redshift $z$ ) of the $\mathrm{SN}$ II supernova rate $R_{S N}(z)$, and on the $\bar{\nu}_{\mathrm{e}}$ energy spectrum $d N / d E$,

$$
\frac{d F}{d E}=\int_{0}^{z_{\max }} \mathrm{R}_{\mathrm{SN}}(z)\left\langle\frac{d N(E(1+z))}{d E}\right\rangle(1+z)\left|\frac{d t}{d z}\right| d z .
$$

The energy of the detected positron, $E_{\mathrm{e}^{+}}$, is related to that of the $\bar{\nu}_{\mathrm{e}}$ by $E_{\mathrm{e}^{+}}=E-1.3 \mathrm{MeV}$. In the above equation the average is over the stellar initial mass function (IMF $\equiv d n_{*} / d \ln M$, where $n_{*}$ is the number of stars of mass $\left.M\right)$. In practice, $\left\langle d N\left(E^{\prime}\right) / d E\right\rangle$ is replaced by $d N\left(E^{\prime}\right) / d E$, with the parameters that determine the SN $\bar{\nu}_{\mathrm{e}}$ spectrum replaced by their respective IMF-averaged values. This approximation is accurate to better than $10 \%$ in the energy window $(>6 \mathrm{MeV}$ ) of interest. To compute $d t / d z$, we use the $\Lambda \mathrm{CDM}$ cosmology (i.e., a flat, cosmological constant dominated model with matter density $\Omega_{M}=0.3$ and Hubble constant $h=0.7)$.

\subsection{Estimating the Supernova Rate}

The core-collapse SN rate at a given redshift is just the star formation rate (SFR) at that redshift for stellar masses larger than $8 M_{\odot}$. To obtain the SN rate we link the SFR to an observable proxy such as the UV or $\mathrm{H} \alpha$ luminosity density. The interpretation of the measured high redshift UV luminosity density is complicated by the fact that UV light is strongly absorbed by dust, while measurements of $\mathrm{H} \alpha$, though less affected by dust, are not as simple to relate to the star formation rate. In addition to correcting for dust extinction, converting the UV light to a SFR requires correcting for the incomplete sampling of the luminosity function, as well as cosmological surface brightness dimming, the latter being true at all frequencies for any extended object [17, 18, 19, 20]

¿From the determination of the SFR we extract the SN rate, $\mathrm{R}_{\mathrm{SN}}(z)=$ $\int_{8 \mathrm{M}_{\odot}}^{30 \mathrm{M}_{\odot}} \dot{\rho}_{*}(z) d n_{*} / d M\left(M^{\prime}\right) d M^{\prime}$, where $\dot{\rho}_{*}(z)$ is the star formation rate in average mass per time per comoving volume. We have limited the upper bound to $30 \mathrm{M}_{\odot}$ due to the potential uncertainties in the neutrino flux from SNe with progenitors more massive than that. Here $d n_{*} / d \ln M$ is the global stellar IMF, assumed constant over redshift, which is a good approximation provided there are no significant correlations between the IMF and 
the environment in which the stars are born. Extant evidence seem to argue against such correlations over the redshift range of interest $(z \lesssim 2)$ [21]. Averaging over a Salpeter IMF for $M>8 \mathrm{M}_{\odot}$, the $\mathrm{SN}$ rate is $\mathrm{R}_{\mathrm{SN}}=\left(\frac{0.013}{\mathrm{M}_{\odot}}\right) \dot{\rho}_{*}$, assuming the star formation rate is measured in solar masses. The conversion factor is not sensitive to the upper limit of the average as long as the upper limit is larger than about $25 \mathrm{M}_{\odot}$. The sensitivity to changes in the IMF slope is more pronounced; if we use Baldry and Glazebrook [22] (BG03; see below) best-fit IMF $\left(d n_{*} / d \ln M \sim M^{-1.15}\right)$, the conversion factor increases by $30 \%$.

Following [23, 24], we parametrize the SFR as $\dot{\rho}_{*}(z) \propto(1+z)^{\beta}$ for $z<1$, and $\dot{\rho}_{*}(z) \propto(1+z)^{\alpha}$ for $z>1$ and use various observational proxies to estimate $\alpha, \beta$, and the normalization. From our assumption above of a redshift-independent IMF, we can then parametrize the $\mathrm{SN}$ rate as

$$
\begin{aligned}
\mathrm{R}_{\mathrm{SN}} & \propto(1+z)^{\beta} \quad \text { for } \quad z<1 \\
\propto(1+z)^{\alpha} & \text { for } \quad z>1 .
\end{aligned}
$$

In Equation 2 we have assumed that the $1<z<2$ behavior continues to higher redshift.

Hogg 23] has compiled measurements of the UV and $\mathrm{H} \alpha$ luminosity density, as well as results from measurement of the near UV emission, far-infrared and radio continuum to obtain the $68 \%$ c.l. limits of $\beta=2.7 \pm 0.7$. Results from the cosmic optical spectrum measurements from the Sloan Digital Sky Survey (SDSS) find limits on $\beta$ from $2-3$ and $\alpha$ from $0-2$ [25] (G03). Using the SDDS data and marginalizing over the IMF and evolution of the SFR, BG03 determine the local density of SFR to be in the range $\dot{\rho}_{*}(0)=(0.5-2.9) \times 10^{-2} \mathrm{M}_{\odot} \mathrm{yr}^{-1} \mathrm{Mpc}^{-3}$. The lower portion of this range for the slopes and normalzation is only valid, however, if the slope of the global IMF is allowed to vary near the BG03 best fit. This lower portion is also consistent with the results of Madau et al. based on the observed UV luminosity density of the whole galaxy population [26]. However, the Madau et al. results do not take into account the correction for surface brightness dimming and provide a smaller average correction for dust extinction [20], and thus permit the possibility that the SN rate may decline for $z \gtrsim 1$. In addition, studies of extra-galactic background light have recently shown that current surface brightness corrections suggest that $\sim 50 \%$ of the high redshift galaxy population is yet unobserved [27].

The choice of how to handle the dust extinction thus dominates the uncertainty budget for the high redshift SN rate. Although the slope of the SFR for $z \gtrsim 1$ is poorly constrained from the SDSS results, examination of high redshift UV data [20] shows that the SFR remains constant or even increases beyond $z \simeq 1$. An increase in $\alpha$ would not significantly change the observable SRN flux at Super-K, as the energy window $\geq 18 \mathrm{MeV}$ is not very sensitive to the $z>1 \mathrm{SN}$ rate. For example, given the parameterization in Eq. 22, we find that the $90 \%$ C.L. Super-K upper bound on the flux of the relic neutrinos of $1.2 \mathrm{~cm}^{-2} \mathrm{~s}^{-1}$ implies that, for $\beta>0.5, \mathrm{R}_{\mathrm{SN}}(0)<2 \times 10^{-4} \mathrm{yr}^{-1} \mathrm{Mpc}^{-3}$, independent of $\alpha$. However, an 


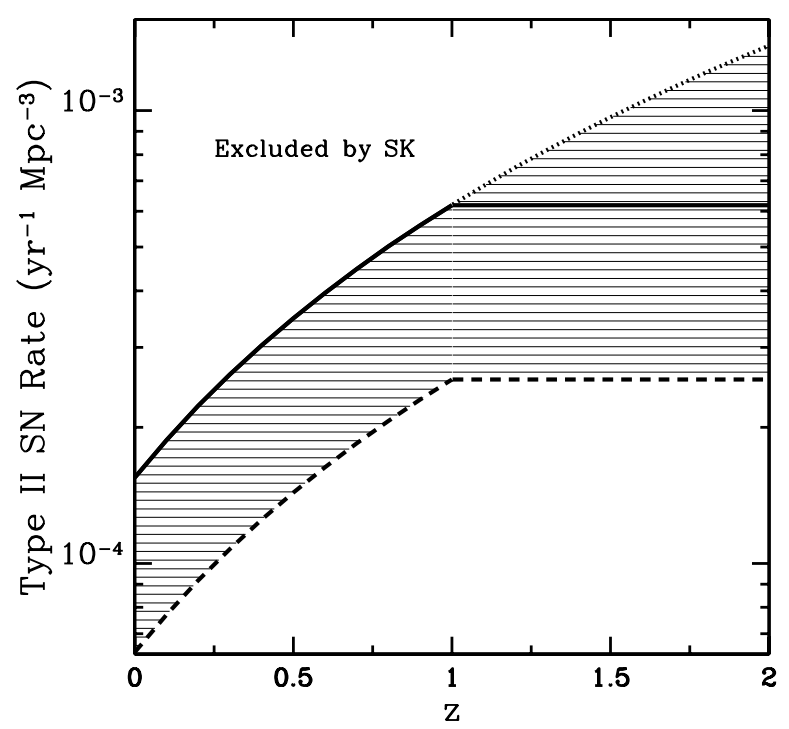

Figure 1. Three representative supernova rates; see eq. (2). The solid curve is based on the median results from UV luminosity density measurements as well as the SDSS cosmic optical spectrum. The dotted curve is an extension of this median model in which the slope of the $z>1 \mathrm{SN}$ rate is increased from $\alpha=0$ to $\alpha=2$; see eq. (2). The area above the shaded region is ruled out by the SK flux limit. The dashed curve is a lower bound to the $\mathrm{SN}$ rate motivated by the SDSS optical spectrum. In each case a Salpeter IMF is used to convert from the SFR to the SN rate.

increase in $\alpha$ would increase the observable flux at KamLAND (or any other relatively low threshold detector such as the proposed SK-GADZOOKS), due to its sensitivity to lower energy neutrinos. Below we discuss the extent to which a comparison of signals between KamLAND and Super-K can constrain the high redshift SFR.

Motivated by the UV density studies as well as the SDSS cosmic optical spectrum, we choose for our "median/best estimate" model $\beta=2.5, \alpha=0, \dot{\rho}_{*}(z=0)=$ $1.6 \times 10^{-2} \mathrm{M}_{\odot} \mathrm{yr}^{-1} \mathrm{Mpc}^{-3}$. This normalization is perfectly consistent with the observations and is specifically chosen to saturate the Super-K result, for the adopted slopes $\beta$ and $\alpha$. This "median" SN rate (and its associated SRN background) is shown by the solid curves in Figures 1 and 2. Note that for this value of $\operatorname{R}_{\mathrm{SN}}(z=0)$, and with an average density of galaxies of $0.01 \mathrm{Mpc}^{-3}$, the present average supernova rate is expected to be $\sim 1$ per 65 years per galaxy. Though the UV and SDSS analyses do permit models with higher star formation rates, such models are ruled out by the Super-K upper limits to the SRN flux. In particular, any models with values of $\beta$ and/or $\mathrm{R}_{\mathrm{SN}}(z=0)$ which exceed our median estimates will violate the Super-K upper bound on the SRN flux. As a specific example, 
if the slopes were fixed at $\beta=3, \alpha=2$, and a Salpeter IMF adopted, this would require a SFR $\dot{\rho}_{*}(z=0)<1.3 \times 10^{-2} \mathrm{M}_{\odot} \mathrm{yr}^{-1} \mathrm{Mpc}^{-3}$ in order to satisfy the Super-K flux limit. Thus, it is clear that the Super-K upper bound on the SRN flux already provides valuable constraints on the cosmic star formation history.

It is interesting, but less certain, to estimate a reasonable lower limit to the SRN flux based on the above observational proxies. We choose a "lower limit" model, shown by the dotted curves in Figures 1 and 2, with $\beta=2, \alpha=0, \dot{\rho}_{*}(z=0)=0.5 \times 10^{-2} \mathrm{M}_{\odot} \mathrm{yr}^{-1} \mathrm{Mpc}^{-3}$. Finally, in order to determine the impact on the SRN flux of increasing the high redshift SFR, we consider a variation to our "median/best estimate" model with $\beta=2.5$ and $\alpha=0$, by setting $\alpha=2$ (dashed curves in Figures 1 and 2). We note that the predictions of Ando, Sato, \& Totani, and of Kawasaki \& Fukugita [5] use SN rates which are closer to this SDSS inspired lower bound.

As already mentioned, with its sensitivity to lower energy neutrinos KamLAND has the potential to probe the high redshift star formation history. About $40 \%$ of the $\bar{\nu}_{e}$ flux in the KamLAND energy window comes from $z>1$; about $10 \%$ of the flux is from SN at $z>2$. Thus it is clear that a comparison of the fluxes at Super-K and KamLAND has the potential to provide valuable information about the star formation history for $1<z<2$. If the SFR at $z \gtrsim 2$ is much larger than the estimates adopted here (there are some hints in this direction [17, 18]), then it might be possible to probe the star formation rate at $z \gtrsim 2$. Below, we quantify the information that can be gleaned about high redshift star formation using Super-K and KamLAND.

\subsection{SRN Spectrum}

The other key ingredient in calculating the background flux of SRN is the spectrum of supernova neutrinos. The supernova neutrino spectrum is typically modelled by a FermiDirac spectrum with an effective chemical potential, $\eta \equiv \mu_{\nu} / T_{\nu}$,

$$
\frac{d N}{d E_{\nu}} \propto \frac{E_{\nu}^{2}}{\exp \left(E_{\nu} / T_{\nu}-\eta\right)+1} .
$$

Equation 3 may be used to relate the average $\bar{\nu}_{\mathrm{e}}$ energy $\left\langle E_{\bar{\nu}_{e}}\right\rangle$ to the $\bar{\nu}_{\mathrm{e}}$ neutrinosphere temperature. Our current understanding of the SN II explosion mechanism (and the observations of neutrinos from SN 1987A [1, 2]) restricts the average energy to the range $14 \lesssim\left\langle E_{\bar{\nu}_{e}}\right\rangle \lesssim 17 \mathrm{MeV}$ (see, e.g., [28]). Relative to a pure Fermi-Dirac spectrum $(\eta=0)$, for a distribution normalized to the total neutrino energy, positive values for $\eta$ correspond to a flattening of the spectrum peak, and an increase in the number of neutrinos in the tail of the distribution. To compromise between the extremes seen in computer simulations, we adopt a Fermi-Dirac distribution with zero chemical potential, characterized by $\left\langle\mathrm{T}_{\bar{\nu}_{\mathrm{e}}}\right\rangle=5 \mathrm{MeV}$ and $\left\langle\mathrm{T}_{\bar{\nu}_{\mu}}\right\rangle=\left\langle\mathrm{T}_{\bar{\nu}_{\tau}}\right\rangle=8 \mathrm{MeV}$. We assume that the total energy carried by each flavor of 
neutrino is $0.5 \times 10^{53} \mathrm{erg}$. The average is taken over the IMF of the $\mathrm{SN}_{\text {II }}$ progenitors. This does not introduce additional significant uncertainties since neither the temperature of the neutrinosphere nor the binding energy of the neutron star depend very sensitively on the the mass of the progenitor and, hence, on the averaging over the IMF. The most recent simulations of Raffelt et al. 229] including muon and tau transport models suggest that the IMF-averaged energies may not be hierarchical, but that $\left\langle E_{\bar{\nu}_{e}}\right\rangle \approx\left\langle E_{\bar{\nu}_{\mu, \tau}}\right\rangle$. For a spectrum normalized to the same total energy output in all neutrino species, such differences do not significantly change the results for the fluxes.

\subsection{Neutrino Mixing}

Neutrino oscillations are of direct relevance to the spectrum of background SRN. If $\bar{\nu}_{\mu}$ and/or $\bar{\nu}_{\tau}$ mix with $\bar{\nu}_{\mathrm{e}}$ the spectrum of the resulting $\bar{\nu}_{\mathrm{e}}$ will be harder. Such higher energy $\bar{\nu}_{\mathrm{e}}$ are easier to detect. From the analyses of solar neutrino and KamLAND data, mixing between the $\nu_{e}\left(\bar{\nu}_{\mathrm{e}}\right)$ and $\nu_{\mu}\left(\bar{\nu}_{\mu}\right)$ flavor eigenstates is favored, with $0.27<\tan ^{2} \theta_{\odot}<0.94$ at $3 \sigma$ [31. In terms of mass eigenstates, the MSW solution implies $\Delta m_{\odot}^{2} \equiv m_{2}^{2}-m_{1}^{2}>0$ [33]. The atmospheric neutrino data [32] are consistent with near-maximal vacuum mixing between the $\nu_{\mu}\left(\bar{\nu}_{\mu}\right)$ and $\nu_{\tau}\left(\bar{\nu}_{\tau}\right)$ flavor eigenstates: $\sin ^{2}(2 \theta)>0.82$ at $90 \%$ C.L. However, unlike the solar neutrino and KamLAND data, the atmospheric neutrino data do not constrain the sign of the corresponding mass-splitting, $\Delta m_{\text {atm }}^{2} \equiv\left|m_{3}^{2}-m_{2}^{2}\right|$. Here, two mass hierarchies are possible; a normal hierarchy, with $m_{3}>m_{2}>m_{1}$, or an inverted hierarchy, with $m_{2}>m_{1}>m_{3}$. As will be noted next, the choice of hierarchy is important for any neutrino mixing inside the supernovae.

Due to the high density inside supernovae, there is the possiblity that before reaching the surface of the supernovae, the neutrinos may have been mixed by the MSW effect 33. In supernovae, there are two MSW resonant density layers, corresponding to the solar and atmospheric mass splittings. Whether the resonant density occurs in the $\nu$ or $\bar{\nu}$ channels depends on the mass hierarchy [34]. For a normal hierarchy, both resonant layers are in the $\nu$ channel. However, the flux that arrives at the surface of the supernova is an incoherent mixture of mass eigenstates. These mass eigenstates travel separately to the surface of the earth and, in the absence of earth matter effects, the final $\bar{\nu}_{\mathrm{e}}$ flux will have a fraction $\sin ^{2} \theta_{\odot}$ of the original $\bar{\nu}_{\mu}$ flux. In contrast, for an inverted hierarchy, the higher density resonant layer is in the $\bar{\nu}$ channel. Deep inside the supernova the $\bar{\nu}_{\mathrm{e}}$ flavor corresponds to the lowest mass eigenstate, whereas in vacuum $\bar{\nu}_{\tau}$ is the lowest mass eigenstate. For adiabatic propagation inside the supernova, and for $\left|\mathrm{U}_{\mathrm{e} 3}\right| \sim 0$ (but not exactly zero) as implied by the reactor neutrino data [15], $\bar{\nu}_{\mathrm{e}}$ remains the lowest mass eigenstate as it exits the supernova, while $\bar{\nu}_{\tau}$ remains the heavy mass eigenstate. The result is that for an inverted hierarchy the $\bar{\nu}_{\mathrm{e}}$ observed on Earth were all "born" as $\bar{\nu}_{\tau}$. 


\section{Results}

Having assembled the necessary ingredients, we are now in a position to calculate the SRN flux at Earth and to estimate the event rates at KamLAND and Super-K. For our standard cases we assume a normal mass hierarchy (no mixing inside the supernovae) and choose $\tan ^{2} \theta_{\odot}=0.46\left(\sin ^{2}\left(\theta_{\odot}\right)=0.31\right)$, in the middle of the solar neutrino and KamLAND range (see $\$ 3.3)$. For Super-K we find

$$
0.3 \lesssim F(E>18 \mathrm{MeV}) \lesssim 1.2 \bar{\nu}_{\mathrm{e}} \mathrm{cm}^{-2} \mathrm{~s}^{-1},
$$

while for KamLAND,

$$
1.9 \lesssim F(E>6 \mathrm{MeV}) \lesssim 7.8 \bar{\nu}_{\mathrm{e}} \mathrm{cm}^{-2} \mathrm{~s}^{-1} .
$$

The upper and lower limits correspond to the solid and dashed curves in Figure 2 respectively. For an inverted mass hierarchy, the detectable flux for $E>18 \mathrm{MeV}$ increases by $\sim 50 \%$, as this interval samples the high-energy tail of the SN neutrino spectrum (locally and, especially, at higher redshifts). If this inverted hierarchy were realized, a lower supernova rate would be required in order to remain consistent with the Super-K upper limit. An inverted hierarchy is less important for the interval $E>6 \mathrm{MeV}$, which is sensitive to more of the (zero-redshift) spectrum, and for our SN neutrino spectrum and SFR parameters there is a negligible change in the detectable rate. Note that the median/best estimate SFR (solid curve in Figure 21) saturates the current Super-K upper bound of $1.2 \bar{\nu}_{\mathrm{e}} \mathrm{cm}^{-2} \mathrm{~s}^{-1}$ [9], suggesting that with a further reduction in the background, Super-K may detect the SRN background (see below for further discussion).

In order to calculate event rates corresponding to these neutrino fluxes, the relevant cross sections and energy thresholds are required. Neglecting the smearing of events due to finite energy resolution, the event rate for positrons produced by the SRN with neutrino energies between $E_{1}$ and $E_{2}$ (assuming $100 \%$ efficiency) is:

$$
\mathrm{R}\left(E_{1}, E_{2}\right)=\mathrm{N}_{\mathrm{p}} \int_{E_{1}+1.3 \mathrm{MeV}}^{E_{2}+1.3 \mathrm{MeV}} \sigma(E) \frac{d F}{d E} d E,
$$

where $E$ is the $\bar{\nu}_{\mathrm{e}}$ energy, $\mathrm{N}_{\mathrm{p}}$ is the number of protons in the fiducial mass of the detector and $\sigma(E)$ is the $\bar{\nu}_{\mathrm{e}}$ cross section on protons [35]. Here we take $E_{2}=82 \mathrm{MeV}$. In the left panel of Figure (2) we show the results for the integral event rates for KamLAND, Super-K, and SK-GADZOOKS. The results for Super-K cover the range of SN rates allowed by combining the SDSS and SK limits, while for KamLAND we show the integral event rate for our best estimate. We estimate a Super-K event rate

$$
1.1 \leq \operatorname{Rate}\left(E_{1}>18 \mathrm{MeV}\right) \leq 3.6 \text { events } / y r,
$$

and a KamLAND event rate

$$
0.1 \leq \operatorname{Rate}\left(E_{1}>6 \mathrm{MeV}\right) \leq 0.4 \text { events } / \text { yr. }
$$


Although KamLAND is a much smaller detector, it does have the advantage of no background and sensitivity to lower energies, permitting it to sample higher redshift supernovae and more of the energy spectrum of lower redshift supernovae. This effect is illustrated in Figure (3) where the fractions of the SRN fluxes contributed by $\mathrm{SN}_{\text {II }}$ at different redshifts are compared for KamLAND $\left(E_{1}=6 \mathrm{MeV}\right)$ and Super-K $\left(E_{1}=18 \mathrm{MeV}\right)$. However, if the SFR has been underestimated at higher redshifts, KamLAND will detect even more relic neutrinos. For example, increasing the slope of the high- $z$ supernova rate from $\alpha=0$ to 2 results in a $\sim 36 \%$ increase in flux for $E_{1}>6 \mathrm{MeV}$, but only a $\sim 4 \%$ change in the event rate for the Super-K window $E_{1}>18 \mathrm{MeV}$.
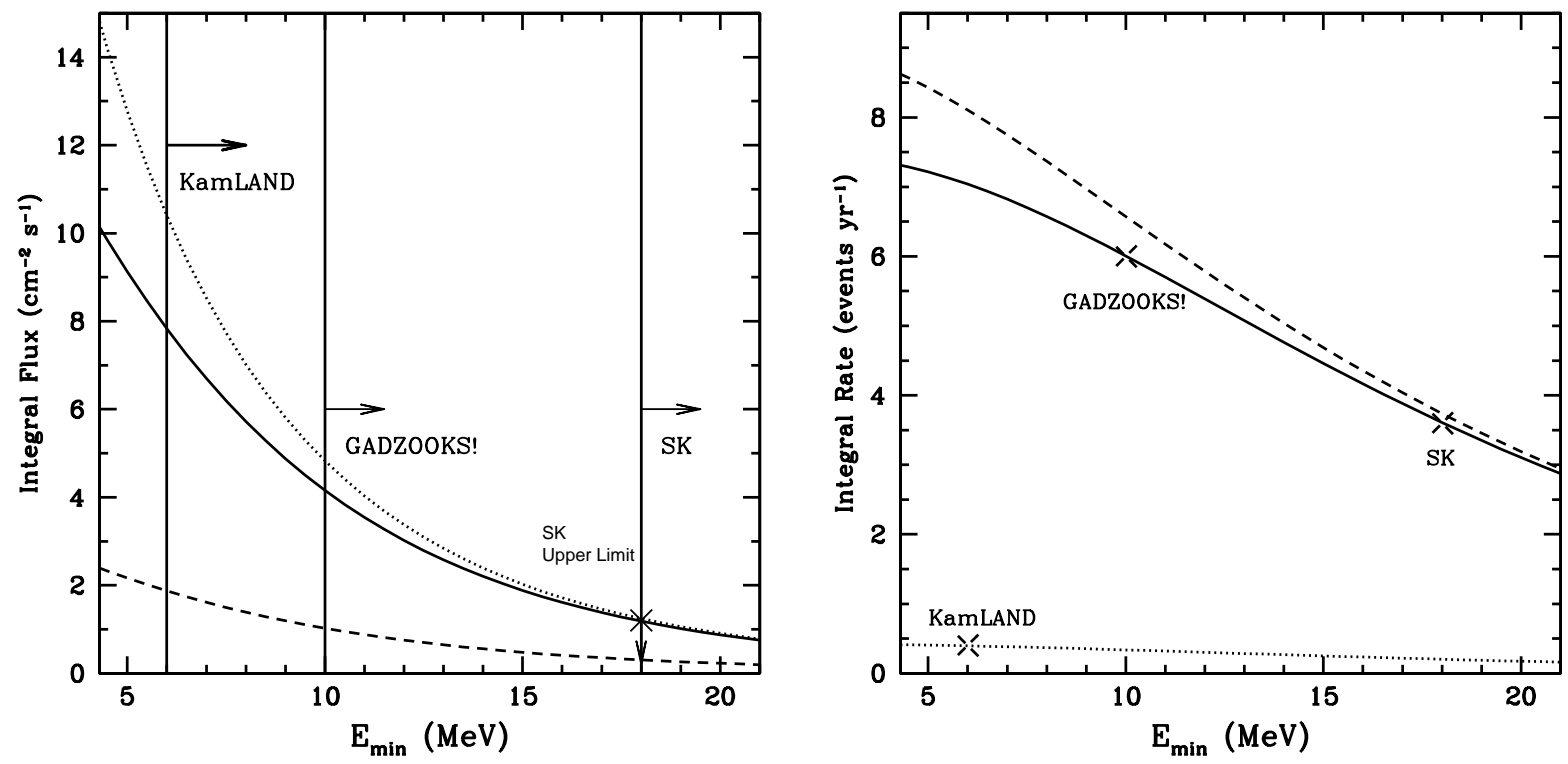

Figure 2. Left: Estimates of the supernova relic $\bar{\nu}_{\mathrm{e}}$ integral flux for positron energies $E>E_{\min }$, as a function of $E_{\min }$. As in Figure 1, the solid curve is our "median SDSS" model with a normalization chosen to saturate the Super-K upper bound. The dotted curve, also saturating the Super-K upper bound, shows the effect of increasing the slope of the $z>1 \mathrm{SN}$ rate. The Super-K upper bound is indicated by the cross. The dashed curve is from an estimated lower bound to the SN rate based on the SDSS results. The Kamland and Super-K threshholds are shown by the vertical lines. Right: Estimates of the $\mathrm{SRN} \bar{\nu}_{\mathrm{e}}$ integral event rates for positron energies $E>E_{\min }$, as a function of $E_{\min }$. The dashed, dotted, and upper solid curves correspond to those for Super-K in Figure 1 The lower solid curve corresponds to the solid curve in Figure 1 for the integral event rate at KamLAND. Crosses mark the levels of the predicted event rates for our best estimate for each detector. 


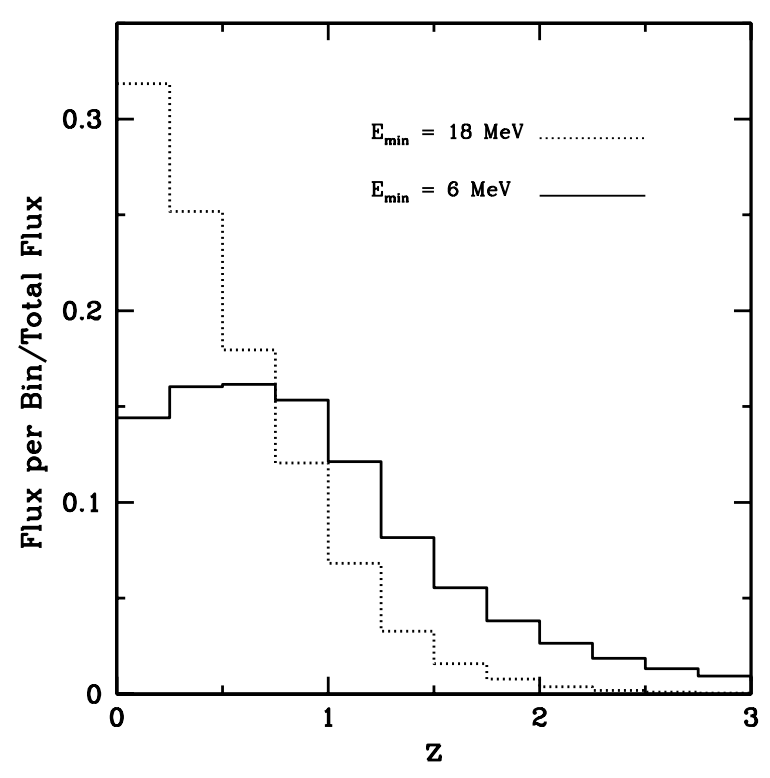

Figure 3. The fraction of the relic neutrino flux contributed by $\mathrm{SN}$ II in each redshift interval. The dotted histogram is for Super-K, while the solid histogram is for KamLAND.

What if both KamLAND and Super-K should detect the SRN? This would open up the possibility of learning about the star formation history for $z \gtrsim 1$, which is currently not well understood. A differential comparison of the KamLAND and Super-K signals, the latter of which comes primarily from $z<1$ (Fig. (3) ), would contain information about the $z>1$ SRN rate and, therefore, about the $z>1$ supernova rate. It is informative to define the quantity

$$
r=\left(\frac{R_{\mathrm{K}} / V_{\mathrm{K}}}{R_{\mathrm{SK}} V_{\mathrm{SK}}}\right)-1,
$$

where $R_{K, S K}$ and $V_{K, S K}$ are the event rates and fiducial volumes at KamLAND (K) and Super-K. The parameter $r$ faithfully captures the effect of changes in $\mathrm{R}_{\mathrm{SN}}(z)$ on the ratio of the flux in the $6-18 \mathrm{MeV}$ window compared to that in the $>18 \mathrm{MeV}$ window. Note that $r$ does not depend on the normalization $\mathrm{R}_{\mathrm{SN}}(0)$; it is only sensitive to the shape of the $\mathrm{R}_{\mathrm{SN}}$ - redshift relation $\mathrm{R}_{\mathrm{SN}}(z)$. Herein lies the sensitivity to supernovae at $z>1$. If the slope of the $z>1$ SFR is increased from $\alpha=0$ to $2, r$ changes by roughly $25 \%$. For example, for the ranges of event rates discussed above, $1.8<r<2.2$. However, changes in $\alpha$ are, to some extent, degenerate with changes in $\beta$. The advantages for studying the evolution of the cosmic SFR provided by the detection of the SRN at KamLAND will come from combining such information with other, more traditional, astronomical observations of the cosmic SFR which can further restrict the range of $\beta$. Such analyses will provide unique 
information about the SFR at high redshift.

\section{Prospects For Detection of the SRN}

If the SRN background is indeed at the level of our median estimate (middle curve of Figure 21), how long will it take for Super-K and KamLAND to detect the SRN? To answer this question we must estimate the size of the error bars on the flux of SRN obtainable at Super$\mathrm{K}$ and KamLAND, as a function of the number of years of data accumulated, $T$. This expected error can then be compared to the likely ranges for the SRN-expected flux. Our model for the error only includes the statistical errors, which in fact is a good approximation 9. The data, given the parameters which determine the SRN and the atmospheric neutrino fluxes, is expected to be Poisson distributed. We neglect the correlations between bins due to energy resolution effects. If the bin sizes are larger than about twice the energy resolution, the correlations will change our results at the $\sim 10 \%$ level.

The recent Super-K analysis [9] lists the detection efficiency for the SRN events as $47 \%$ for $E_{1} \leq 34 \mathrm{MeV}$ and $79 \%$ for $E_{1}>34 \mathrm{MeV}$ after full data reduction. We use an efficiency of unity for Kamland. We modify the event rate for the SRN and the backgrounds in accordance with this prescription for efficiency. The event rate in each energy bin is denoted by $S_{\alpha i}$ where $i$ refers to the energy bin. $\alpha$ identifies whether the event rate is due to the SRN, invisible muon decay, or the atmospheric neutrinos; $f_{\alpha}$ is the corresponding flux normalization. Assume that each bin accumulates $N_{i}$ events (after full data reduction) in $T$ years of runtime. The Poisson measurement error is $\sigma_{i}^{2}=N_{i}$. We define

$$
F(\alpha, \beta)=T \Sigma_{i} \frac{T}{N_{i}} \frac{S_{\alpha i}}{f_{\alpha}} \frac{S_{\beta i}}{f_{\beta}} .
$$

The error on $f_{\alpha}$ is then given by $\left[F^{-1}(\alpha, \alpha)\right]^{1 / 2}$. Assuming the model described above is a good fit to data (as demonstrated by the Super-K results [9]), we forecast the errors on $f_{\alpha}$ by replacing $N_{i}$ by its expected mean value, $T \Sigma_{\alpha} S_{\alpha i}$.

With the above efficiency corrections, for the Super-K window $E_{1}>18 \mathrm{MeV}$ the expected 1- $\sigma$ error on the SRN event rate with $T$ years of data is $\sim 11 / \sqrt{T}$ events/yr. We note that with a total of $8-9$ years of data, Super-K will be able to make a 1- $\sigma$ detection if the SRN flux is close to our estimate from median SFR parameters (solid line in Figure 2). For KamLAND, the primary background for the SRN is from atmospheric $\nu_{e}\left(\bar{\nu}_{\mathrm{e}}\right)$, which has negligible expected rate in the range $\lesssim 25 \mathrm{MeV}$. To determine this background at KamLAND, we correct the results from Super-K [13] for the number of target protons in the 1 kton KamLAND volume. We determine that KamLAND can make a 1- $\sigma$ detection of the SRN flux with $\sim 5$ kton-years of data, if the flux is close to the solid curve in Figure 2. 
These estimated times to detection are long because the expected signal is close to the background when the detector is big and high threshold (Super-K) or small and low background (KamLAND). We note that if Super-K can sufficiently reduce the background from spallation and sub-Cerenkov $\mu^{ \pm}$decays, it can become an optimal SRN detector, opening up the $E>10 \mathrm{MeV}$ window for detection of the SRN [4]. Assuming $80 \%$ efficiency for detecting the SRN for $E>10 \mathrm{MeV}$ (M. Vagins, private communication), and a factor of 5 reduction in the backgrounds [4], we determine that Super-K will make an immediate detection of the SRN background, at minimum a 1- $\sigma$ detection with less than one year of data. This opens up the possibility that we could constrain the space of fundamental $\mathrm{SN}$ II parameters which affect the spectrum of neutrino flux from a SN II significantly. The general strategy would be to relax some assumption (motivated by simulations) about the neutrino spectrum and test them with SRN data.

\section{Conclusions}

Stimulated by the Super-K collaboration's 9] remarkable reduction in their backgrounds for detection of the SN relic neutrinos $(E \geq 18 \mathrm{MeV})$, by the absence of significant backgrounds to the SRN detection at KamLAND [7] (for $E>6 \mathrm{MeV}$ ), and by recent progress in pinning down the cosmic star formation history from new observations including the Sloan Digital Sky Survey, we have calculated the expected fluxes and event rates at Super-K and KamLAND. The SRN flux presented here, based on the SN rate derived from the star formation data, saturates the current Super-K upper bound, suggesting that Super$\mathrm{K}$ may be close to observing the SRN. For our median model (see Fig. 2), we conclude that the predicted flux at Super-K saturates the current experimental upper limit, with a corresponding event rate of $3.6 \mathrm{yr}^{-1}$. In this case Super-K will be able to make a $1-\sigma$ detection by doubling their data set, i.e. with a total of about 9 years (including the existing 4 years of data). If Super-K does see the SRN background flux, assuming that KamLAND is $100 \%$ efficient and can use the entire $1 \mathrm{kton}$ volume for detection of the SRN, the associated event rate expected at KamLAND is small, $\sim 0.4 \mathrm{yr}^{-1}$. However, in this ideal scenario, the lack of any significant competing backgrounds will permit a 1- $\sigma$ detection of the SRN with only 5 years of data.

\section{Acknowlegments}

G.S. thanks John Learned for encouragement and several discussions which helped to stimulate this investigation. We thank John Beacom and Mark Vagins for discussions about the supernova relic neutrinos and their GADZOOKS proposal. We thank Sinichiro Ando for correcting an equation in an earlier draft of this paper. The research of G.S., L.S., and 
T.P.W. are supported at OSU by the Department of Energy grant DE-FG02-91ER40690; M.K. is supported at U. C. Davis from the NSF and from NASA grant NAG5-11098 .

\section{References}

[1] Hirata K, et al. , 1987 Phys. Rev. Lett. 581490

[2] Bionta R M, et al. , 1987 Phys. Rev. Lett. 581494

[3] Davis M, et al. , Discoveries and Research Prospects from 6-to 10-Meter-Class Telescopes II. Edited by P. Guhathakurta. Proceedings of the SPIE, Volume 4834, p 161

[4] Beacom J F and Vagins M R, 2003 Preprint hep-ph/0309300

[5] Bisnovatyi-Kogan G S and Seidov Z F, 1984 Sov. Astron. 26 132; Krauss L M, Glashow S L, and Schramm D N, 1984 Nature 310, 191; Dar A , 1985 Phys. Rev. Lett. 55, 1422; Woosley S E, Wilson J R, and Mayle R, 1986 Astrophys. J. 302 19; Totsuka Y, Rep. Prog. Phys. 199255 377; Totani T and Sato K, 1995 Astropart. Phys. 3 367; Totani T, Sato K, and Yoshii Y, 1996 Astrophys. J. 460 303; Malaney R A, 1997 Astropart. Phys. 7 125; Hartmann D H and Woosley S E, 1997 Astropart. Phys. 7 137; Ando S, Sato K, and Totani T, 2003 Astropart. Phys. 18 307; Fukugita M and Kawasaki M, 2003 Mon. Not. Roy. Astron. Soc. 340 L7; Ando S and Sato K, 2003 Phys. Lett. B 559113

[6] Kaplinghat M, Steigman G, and Walker T P, 2000 Phys. Rev. D 62, 043001 (KSW)

[7] (The KamLAND Collaboration), Eguchi K, et al. 2003 Phys. Rev. Lett. 90, 021802

[8] (The SNO Collaboration), Ahmad Q R et al. 2002 Phys. Rev. Lett. 89, 011301

[9] (The Super-Kamiokande Collaboration), Malek M et al. , 2003 Phys. Rev. Lett. 90061101

[10] Zhang W, et al. , 1988 Phys. Rev. Lett. 61385

[11] Shiozawa M, talk at the International Workshop on a Next Generation Long-Baseline Neutrino Oscillation Experiment, Tsukuba, Japan, May 2001; http://neutrino.kek.jp/jhfnu/workshop2/ohp.html Jung C K, Preprint hep-ex/0005046 Casper D, talk at New Initiatives in Lepton Flavor Violation and Neutrino Oscillations with Very Intense Muon and Neutrino Sources, Honolulu, October 2000; Barger V, Marfatia D, and Wood B P, 2002 Phys. Lett. B 54737

[12] (The Super-Kamiokande Collaboration), Gando Y et al. , 2003 Phys. Rev. Lett. 90171302

[13] Gaisser T K and Stanev T, 1988 Phys. Rev. D 3885

Honda M, Kajuta T, Kasahara K, and Midorikawa S, 1995 Phys. Rev. D 524985

[14] Alivisatos P, et al., 1998, KAMLAND: a liquid scintillator Anti-Neutrino Detector at the Kamioka Site, preprint Stanford-HEP-98-03, Tohoku-RCNS-98-15;

[15] Bemporad C, Gratta G, and Vogel P, 2002 Rev. Mod. Phys. 74297

[16] The KamLAND Collaboration, 2003 Preprint hep-ex/0310047

[17] Lanzetta K M, Yahata N, Pascarelle S, Chen H, and Fernandez-Soto A, 2002 Astrophys. J. 570492

[18] Conti A et al. 2003, Astron.J. 126, 2330

[19] Rowan-Robinson M, 2003 Mon. Not. Roy. Astron. Soc. 345819

[20] Thompson R I, 2003 Astrophys.J. 596748

[21] Scalo J M, 1998 The Stellar Initial Mass Function, ed. G. Gilmore and D. Howell (San Francisco: ASP), p 201

[22] Baldry I K and Glazebrook K, 2003 Astron.J. 1261483 (BG03)

[23] Hogg D W, 2001 Preprint astro-ph/0105280

[24] Baldry I K et al., 2002 Astrophys. J. 569, 582

[25] Glazebrook K et al., 2003 Astrophys.J. 58755 (G03)

[26] Madau P, Pozzetti L, and Dickinson M, 1998 Astrophys. J. 498106 
[27] Bernstein R A, Freedman W L, \& Madore B F, 2002 Astrophys.J. 571 107; Nagamime K et al., astro-ph/0311294

[28] Raffelt G, Supernova Neutrinos 1996 Stars as Laboratories of Fundamental Physics, (The University of Chicago Press, Chicago); Myra E S and Burrows A, 1990 Astrophys. J. 364 222; Janka H T and Hillebrandt W, 1989 Astron. and Astrophys. 224 49; Myra E S et al., 1988 Proc. in Supernova 1987 A in the Large Magellanic Cloud, ed M Kafatos and A Michalitsianos, (Cambridge University Press, Cambridge) p 213; Suzuki H, Supernova Neutrinos, 1994 Physics and Astrophysics of Neutrinos, ed M Fukugita and A Suzuki (Springer-Verlag, Berlin) p 763

[29] Raffelt G et al. 2003 Contributions to Proc. NOON 2003: The 4th Workshop on Neutrino Oscillations and their Origin. astro-ph/0303226

[30] Apollonio M et al., 1999 Phys. Lett. B 466415

[31] Bahcall J N, Gonzalez-Garcia M C, and Pena-Garay C, 2003 JHEP 0302 009; Bahcall J N and Pena-Garay C, 2003 JHEP 0311004

[32] (The Super-Kamiokande Collaboration), Fukuda Y et al. , 1998 Phys. Rev. Lett. 811562

[33] Wolfenstein L, 1978 Phys. Rev. D 17 2369; Mikheyev S P and Smirnov A Y, 1985 Sov. J. Nucl. Phys. 42, 913

[34] Dighe A S and Smirnov A Y, 2000 Phys. Rev. D 62033007

[35] Vogel P and Beacom J F, 1999 Phys. Rev. D 60053003 